\begin{tabular}{|c|l|}
\hline Title & Boundary behavior of solutions of the Helmholtz equation \\
\hline Author(s) & Hirata, Kentaro \\
\hline Citation & Hokkaido University Preprint Series in Mathematics, 810, 1-8 \\
\hline Issue Date & 2006 \\
\hline DOI & 10.14943/83960 \\
\hline Doc URL & http://hdl.handle.net/2115/69618 \\
\hline Type & bulletin (article) \\
\hline File Information & pre810.pdf \\
\hline
\end{tabular}

Instructions for use 


\title{
BOUNDARY BEHAVIOR OF SOLUTIONS OF THE HELMHOLTZ EQUATION
}

\author{
KENTARO HIRATA
}

\begin{abstract}
This paper is concerned with the boundary behavior of solutions of the Helmholtz equation in $\mathbb{R}^{n}$. In particular, we give a Littlewood-type theorem to show that the approach region introduced by Korányi and Taylor (1983) is best possible.
\end{abstract}

\section{INTRODUCTION}

Let $n \geq 2$ and let us denote a typical point in $\mathbb{R}^{n}$ by $x=\left(x_{1}, \ldots, x_{n}\right)$. The usual inner product and norm are written respectively as $\langle x, y\rangle=x_{1} y_{1}+\cdots+x_{n} y_{n}$ and $|x|=\sqrt{\langle x, x\rangle}$. The symbol $O(n)$ stands for the set of all orthogonal transformations on $\mathbb{R}^{n}$. Let $\lambda>0$. We consider the Helmholtz equation

$$
\Delta u=\lambda^{2} u \quad \text { in } \mathbb{R}^{n},
$$

where $\Delta=\partial^{2} / \partial x_{1}^{2}+\cdots+\partial^{2} / \partial x_{n}^{2}$. It is known that the Martin boundary for positive solutions of (1.1) can be identified with the unit sphere $S$ of $\mathbb{R}^{n}$, and that every positive solution $u$ of (1.1) can be represented as $u=K \mu$ for some Radon measure $\mu$ on $S$, where

$$
K \mu(x)=\int_{S} e^{\lambda\langle x, y\rangle} d \mu(y) \quad \text { for } x \in \mathbb{R}^{n} .
$$

See [4, Corollary to Theorem 4] and [9]. Let $\sigma$ denote the surface measure on $S$. Since $K \sigma(x) \rightarrow+\infty$ as $x \rightarrow \infty$ (cf. Lemma 2.1), we investigate the behavior at infinity of the normalization $K \mu / K \sigma$. Let $e=(1,0, \ldots, 0)$ and let $\Omega$ be an unbounded subset of $\mathbb{R}^{n}$ converging to $e$ at $\infty$ in the sense that $|x /| x|-e| \rightarrow 0$ as $x \rightarrow \infty$ within $\Omega$. We write $\Omega(y)$ for the image of $\Omega$ under an element of $O(n)$ mapping $e$ to $y$. Then $\{\Omega(y): y \in S\}$ makes a collection of approach regions. By the notation $\Omega(y) \ni x \rightarrow \infty$, we mean that $x \rightarrow \infty$ within $\Omega(y)$. Korányi and Taylor [9] considered the following approach region. For $\alpha>0$ and $y \in S$, define

$$
\mathcal{A}_{\alpha}(y)=\left\{x \in \mathbb{R}^{n}:|x-| x|y| \leq \alpha \sqrt{|x|}\right\} .
$$

Theorem A. Let $\alpha>0$ and let $\mu$ be a Radon measure on $S$. Then

$$
\lim _{\mathcal{A}_{\alpha}(y) \ni x \rightarrow \infty} \frac{K \mu}{K \sigma}(x)=\frac{d \mu}{d \sigma}(y) \quad \text { for } \sigma \text {-a.e. } y \in S \text {. }
$$

2000 Mathematics Subject Classification. 31B25, 35J05.

Key words and phrases. boundary behavior, Helmholtz equation. 
This result corresponds to Fatou's theorem [5] for the boundary behavior of harmonic functions in the unit ball or the upper half space of $\mathbb{R}^{n}$ (see also $[8,12]$ for invariant harmonic functions in the unit ball of $\mathbb{C}^{n}$ ). The result corresponding to Nagel-Stein's theorem [11] was established by Berman and Singman [3]. The potential theoretic extension was due to Gowrisankaran and Singman [6]. These results show that there exists an unbounded subset $\Omega$ of $\mathbb{R}^{n}$ converging to $e$ at $\infty$ such that

$$
\limsup _{\Omega \ni x \rightarrow \infty} \frac{|x-| x|e|}{\sqrt{|x|}}=+\infty
$$

and that

$$
\lim _{\Omega(y) \ni x \rightarrow \infty} \frac{K \mu}{K \sigma}(x)=\frac{d \mu}{d \sigma}(y) \quad \text { for } \sigma \text {-a.e. } y \in S,
$$

whenever $\mu$ is a Radon measure on $S$. Berman and Singman also showed the following (see [3, Theorem B and Remark 1. 13(a)]).

Theorem B. Let $\Omega$ be an unbounded subset of $\mathbb{R}^{n}$ converging to e at $\infty$ and satisfying

$$
\limsup _{\Omega \ni x \rightarrow \infty} \frac{|x-| x|e|}{\sqrt{|x|}}=+\infty .
$$

Suppose in addition that $\Omega$ is invariant under all elements of $O(n)$ that preserve the point $e$. Then there exists a Radon measure $\mu$ on $S$ such that

$$
\limsup _{\Omega(y) \ni x \rightarrow \infty} \frac{K \mu}{K \sigma}(x)=+\infty \quad \text { for every } y \in S .
$$

Note that the second assumption on $\Omega$ can not be omitted from their construction even if "lim sup" in (1.3) is replaced by "lim".

The purpose of this paper is to show the following Littlewood-type theorem. See [10, 1, $2,7]$ for harmonic or invariant harmonic functions.

Theorem 1.1. Let $\gamma$ be a curve in $\mathbb{R}^{n}$ converging to e at $\infty$ and satisfying

$$
\lim _{\gamma \ni x \rightarrow \infty} \frac{|x-| x|e|}{\sqrt{|x|}}=+\infty \text {. }
$$

Then there exists a solution $u$ of (1.1) such that $u / K \sigma$ is bounded in $\mathbb{R}^{n}$ and that $u / K \sigma$ admits no limits as $x \rightarrow \infty$ along $T \gamma$ for every $T \in O(n)$.

Remark 1.2. We indeed construct $u$ satisfying $-1 \leq u / K \sigma \leq 1$ and

$$
\liminf _{T \gamma \ni x \rightarrow \infty} \frac{u}{K \sigma}(x)=-1 \text { and } \quad \limsup _{T \gamma \ni x \rightarrow \infty} \frac{u}{K \sigma}(x)=1
$$

for every $T \in O(n)$. Note that "lim" in (1.4) can not be replaced by "lim sup" as mentioned above (cf. $[3,6])$.

The proof of Theorem 1.1 is based on our previous work [7] for invariant harmonic functions in the unit ball of $\mathbb{C}^{n}$, which was a refinement of Aikawa's method $[1,2]$ for harmonic functions in the unit disc or the upper half space of $\mathbb{R}^{n}$. In Section 4, we remark that our construction and estimates are applicable to show Theorem B. 


\section{LEMMAS}

The symbol $A$ denotes an absolute positive constant depending only on $\lambda$ and the dimension $n$, and may change from line to line. The following estimate is found in [3, Lemma 4.1].

Lemma 2.1. There exists a constant $A>1$ such that

$$
\frac{1}{A} e^{\lambda|x|}|x|^{(1-n) / 2} \leq K \sigma(x) \leq A e^{\lambda|x|}|x|^{(1-n) / 2}
$$

whenever $|x| \geq 1$.

The surface ball of center $y \in S$ and radius $r>0$ is denoted by

$$
Q(y, r)=\{x \in S:|x-y|<r\} .
$$

Then we observe that

$$
\lim _{r \rightarrow 0} \frac{\sigma(Q(y, r))}{r^{n-1}}=\nu_{n-1}
$$

where $\nu_{n-1}$ is the volume of the unit ball of $\mathbb{R}^{n-1}$. Moreover, there exists a constant $A>1$ such that

$$
\frac{1}{A} r^{n-1} \leq \sigma(Q(y, r)) \leq A r^{n-1} \text { for } 0<r \leq 2 .
$$

Let $\pi$ be the radial projection onto $S$, i.e., $\pi(x)=x /|x|$ for $x \in \mathbb{R}^{n} \backslash\{0\}$. For a Radon measure $\mu$ on $S$, we define the maximal function $M_{(c)} \mu$ with parameter $c \geq 1$ by

$$
M_{(c)} \mu(x)=\sup \left\{\frac{\mu(Q(\pi(x), r))}{r^{n-1}}: r \geq \frac{c}{\sqrt{|x|}}\right\} .
$$

Lemma 2.2. Let $c \geq 1$ and let $\mu$ be a Radon measure on $S$. Then

$$
\frac{K \mu}{K \sigma}(x) \leq A\left(|x|^{(n-1) / 2} \mu(Q(\pi(x), c / \sqrt{|x|}))+\frac{1}{c} M_{(c)} \mu(x)\right)
$$

whenever $|x| \geq 1$.

Proof. Let $|x| \geq 1$. Since $|x|-\langle x, y\rangle=|x||\pi(x)-y|^{2} / 2$ for $y \in S$, it follows from Lemma 2.1 that

$$
\frac{K \mu}{K \sigma}(x) \leq A|x|^{(n-1) / 2} \int_{S} e^{-(\lambda / 2)|x||\pi(x)-y|^{2}} d \mu(y) .
$$

Let $Q_{1}=Q(\pi(x), c / \sqrt{|x|})$ and $Q_{j}=Q(\pi(x), j c / \sqrt{|x|}) \backslash Q(\pi(x),(j-1) c / \sqrt{|x|})$ for $j=$ $2, \ldots, N$, where $N$ is the smallest integer such that $N c / \sqrt{|x|}>2$. Then, for $j=1, \ldots, N$,

$$
\int_{Q_{j}} e^{-(\lambda / 2)|x||\pi(x)-y|^{2}} d \mu(y) \leq e^{-(\lambda / 2)((j-1) c)^{2}} \mu(Q(\pi(x), j c / \sqrt{|x|})) .
$$

Therefore the right hand side of (2.3) is bounded by

$$
A\left(|x|^{(n-1) / 2} \mu(Q(\pi(x), c / \sqrt{|x|}))+\sum_{j \geq 2} e^{-(\lambda / 2)((j-1) c)^{2}}(j c)^{n-1} M_{(c)} \mu(x)\right) .
$$


Since $\sum_{j \geq 2} e^{-(\lambda / 2)((j-1) c)^{2}}(j c)^{n-1} \leq A / c$, we obtain the required estimate.

For an integrable function $f$ on $S$, we write $K f=K(f d \sigma)$ and $M_{(c)} f=M_{(c)}(|f| d \sigma)$.

Lemma 2.3. The following statements hold.

(i) Let $\mu$ be a Radon measure on $S$. Then

$$
\frac{K \mu}{K \sigma}(x) \leq A M_{(1)} \mu(x)
$$

whenever $|x| \geq 1$.

(ii) Let $y \in S, 0<r<1$ and $c \geq 1$. Suppose that $f$ is a Borel measurable function on $S$ such that $f=1$ on $Q(y, c r)$ and $|f| \leq 1$ on $S$. Then

whenever $\sqrt{t} \geq 1 / r$.

$$
\frac{K f}{K \sigma}(t y) \geq 1-\frac{A}{c}
$$

Proof. Lemma 2.2 with $c=1$ gives (i). To show (ii), let $g=(1-f) / 2$. Then $g=0$ on $Q(y, c r)$ and $|g| \leq 1$ on $S$. Observe from Lemma 2.2 and (2.2) that if $\sqrt{t} \geq 1 / r$, then

$$
\frac{K g}{K \sigma}(t y) \leq \frac{A}{c} M_{(c)} g(t y) \leq \frac{A}{c} \sup \left\{\frac{\sigma(Q(y, \rho))}{\rho^{n-1}}: \rho \geq \frac{c}{\sqrt{t}}\right\} \leq \frac{A}{c} .
$$

Since $K f=K \sigma-2 K g$, we obtain (ii).

For a set $E$, let $\operatorname{diam} E=\sup \{|x-y|: x, y \in E\}$.

Lemma 2.4. Let $\gamma$ be a curve in $\mathbb{R}^{n}$ converging to e at $\infty$ and satisfying (1.4). Then there exist sequences of numbers $\left\{a_{j}\right\}_{j \geq 1},\left\{b_{j}\right\}_{j \geq 1}$ and subarcs $\left\{\gamma_{j}\right\}_{j \geq 1}$ of $\gamma$ with the following properties:

(i) $1<a_{1}<b_{1}<\cdots<a_{j}<b_{j}<a_{j+1}<b_{j+1}<\cdots \rightarrow+\infty$,

(ii) $a_{j} \leq \sqrt{|x|} \leq b_{j}$ for $x \in \gamma_{j}$,

(iii) $b_{j-1} \operatorname{diam} \pi\left(\gamma_{j}\right) \leq 1$ if $j \geq 2$,

(iv) $\lim _{j \rightarrow+\infty} a_{j} \operatorname{diam} \pi\left(\gamma_{j}\right)=+\infty$.

Proof. Let $\left\{\alpha_{j}\right\}$ be a sequence such that $\alpha_{j} \rightarrow+\infty$ as $j \rightarrow+\infty$, and let us choose $\left\{a_{j}\right\}$, $\left\{b_{j}\right\}$ and $\left\{\gamma_{j}\right\}$ inductively. By (1.4), we find $a_{1}>\max \left\{1, \inf _{x \in \gamma} \sqrt{|x|}\right\}$ with

$$
\sqrt{|x|}|\pi(x)-e| \geq \alpha_{1} \text { for } x \in \gamma \cap\left\{\sqrt{|x|} \geq a_{1}\right\} .
$$

Let $\gamma^{\prime}$ be the connected component of $\gamma \cap\left\{\sqrt{|x|} \geq a_{1}\right\}$ which converges to $\infty$, and let $x_{1} \in \gamma^{\prime} \cap\left\{\sqrt{|x|}=a_{1}\right\}$. Then

$$
\operatorname{diam} \pi\left(\gamma^{\prime}\right) \geq\left|\pi\left(x_{1}\right)-e\right| \geq \frac{\alpha_{1}}{a_{1}} .
$$

Let $\gamma^{\prime \prime}$ be a subarc of $\gamma^{\prime}$ starting from $x_{1}$ toward $\infty$ such that

$$
\sup _{x \in \gamma^{\prime \prime}} \sqrt{|x|}<+\infty \quad \text { and } \quad \operatorname{diam} \pi\left(\gamma^{\prime \prime}\right) \geq \frac{1}{2} \operatorname{diam} \pi\left(\gamma^{\prime}\right) .
$$


We take $b_{1}>\sup _{x \in \gamma^{\prime \prime}} \sqrt{|x|}$. Let $\gamma_{1}$ be the connected component of $\gamma \cap\left\{a_{1} \leq \sqrt{|x|} \leq b_{1}\right\}$ containing $\gamma^{\prime \prime}$. Then

$$
\operatorname{diam} \pi\left(\gamma_{1}\right) \geq \frac{\alpha_{1}}{2 a_{1}} .
$$

We next choose $a_{2}, b_{2}$ and $\gamma_{2}$ as follows. By (1.4) and the fact that $|\pi(x)-e| \rightarrow 0$ as $x \rightarrow \infty$ along $\gamma$, we find $a_{2}>b_{1}$ such that

$$
\frac{1}{2 b_{1}} \geq|\pi(x)-e| \geq \frac{\alpha_{2}}{\sqrt{|x|}} \quad \text { for } x \in \gamma \cap\left\{\sqrt{|x|} \geq a_{2}\right\} \text {. }
$$

Repeat the above process to get $b_{2}>a_{2}$ and $\gamma_{2}$ such that $a_{2} \leq \sqrt{|x|} \leq b_{2}$ for $x \in \gamma_{2}$ and $\operatorname{diam} \pi\left(\gamma_{2}\right) \geq \alpha_{2} / 2 a_{2}$. Then (2.4) also yields that

$$
\operatorname{diam} \pi\left(\gamma_{2}\right) \leq 2 \sup _{x \in \gamma_{2}}|\pi(x)-e| \leq \frac{1}{b_{1}} .
$$

Continue this process to obtain the required sequences.

\section{Construction}

Throughout this section, we suppose that $\left\{a_{j}\right\}_{j \geq 1},\left\{b_{j}\right\}_{j \geq 1}$ and $\left\{\gamma_{j}\right\}_{j \geq 1}$ are as in Lemma 2.4. Let

$$
\ell_{j}=\frac{\operatorname{diam} \pi\left(\gamma_{j}\right)}{3}, \quad c_{j}=\sqrt{a_{j} \operatorname{diam} \pi\left(\gamma_{j}\right)} \quad \text { and } \quad \rho_{j}=\frac{c_{j}}{a_{j}} .
$$

Then, by Lemma 2.4,

$$
\lim _{j \rightarrow+\infty} \ell_{j}=0, \quad \lim _{j \rightarrow+\infty} \frac{\rho_{j}}{\ell_{j}}=0 \quad \text { and } \quad \lim _{j \rightarrow+\infty} c_{j}=+\infty .
$$

Therefore, in the construction below, we may assume that $\rho_{j}<\ell_{j}$ for every $j \in \mathbb{N}$. For each $j \in \mathbb{N}$, we choose finitely many points $\left\{y_{j}^{\nu}\right\}_{\nu}$ in $S$ such that

(I) $S=\bigcup_{\nu} Q\left(y_{j}^{\nu}, \ell_{j}\right)$,

(II) $Q\left(y_{j}^{\mu}, \ell_{j} / 2\right) \cap Q\left(y_{j}^{\nu}, \ell_{j} / 2\right)=\emptyset$ if $\mu \neq \nu$.

We define

$$
\begin{aligned}
& M_{j}=\bigcup_{\nu}\left\{y \in S:\left|y-y_{j}^{\nu}\right|=\ell_{j}\right\}, \\
& G_{j}=\left\{x \in \mathbb{R}^{n}: a_{j} \leq \sqrt{|x|} \leq b_{j} \text { and } \pi(x) \in M_{j}\right\} .
\end{aligned}
$$

Then we have the following.

Lemma 3.1. $T \gamma_{j} \cap G_{j} \neq \emptyset$ for any $T \in O(n)$ and $j \in \mathbb{N}$.

Proof. By (I), we find $\nu$ with $\pi\left(T \gamma_{j}\right) \cap Q\left(y_{j}^{\nu}, \ell_{j}\right) \neq \emptyset$. Since $\operatorname{diam} \pi\left(T \gamma_{j}\right)=\operatorname{diam} \pi\left(\gamma_{j}\right)=$ $3 \ell_{j}$, we see that $\pi\left(T \gamma_{j}\right) \cap M_{j} \neq \emptyset$. Therefore it follows from $T \gamma_{j} \subset\left\{a_{j} \leq \sqrt{|x|} \leq b_{j}\right\}$ that $T \gamma_{j} \cap G_{j} \neq \emptyset$. 
Let $R_{j}^{\nu}=\left\{y \in S: \ell_{j}-\rho_{j}<\left|y-y_{j}^{\nu}\right|<\ell_{j}+\rho_{j}\right\}$ and define

$$
E_{j}=\bigcup_{\nu} R_{j}^{\nu}
$$

Note that $Q\left(y, \rho_{j}\right) \subset E_{j}$ if $y \in M_{j}$. By $\mathcal{X}_{E}$ we denote the characteristic function of $E$.

Lemma 3.2. The following properties for the above $\left\{E_{j}\right\}_{j \geq 1}$ hold.

(i) $\lim _{j \rightarrow+\infty}\left(\sup \left\{\frac{K \mathcal{X}_{E_{j}}}{K \sigma}(x): \sqrt{|x|} \leq b_{j-1}\right\}\right)=0$.

(ii) $\lim _{j \rightarrow+\infty} \sigma\left(E_{j}\right)=0$.

Proof. Since the value $\sigma\left(R_{j}^{\nu}\right)$ is independent of $\nu$, we write $\sigma_{j}=\sigma\left(R_{j}^{\nu}\right)$. For a moment, we fix $j$ and let $\sqrt{|x|} \leq b_{j-1}$. By Lemma 2.3(i),

$$
\begin{aligned}
\frac{K \mathcal{X}_{E_{j}}}{K \sigma}(x) & \leq A M_{(1)} \mathcal{X}_{E_{j}}(x) \\
& \leq A \sup \left\{\sum_{\nu} \frac{\sigma\left(R_{j}^{\nu} \cap Q(\pi(x), r)\right)}{r^{n-1}}: r \geq \frac{1}{\sqrt{|x|}}\right\} \\
& \leq A \sup \left\{\frac{\sigma_{j}}{r^{n-1}} N_{j}: r \geq \frac{1}{\sqrt{|x|}}\right\},
\end{aligned}
$$

where $N_{j}$ is the number of $\nu$ such that $R_{j}^{\nu} \cap Q(\pi(x), r) \neq \emptyset$. If $r \geq 1 / \sqrt{|x|}$, then $r \geq$ $1 / b_{j-1} \geq \operatorname{diam} \pi\left(\gamma_{j}\right)=3 \ell_{j}$ by Lemma 2.4. Therefore $R_{j}^{\nu} \cap Q(\pi(x), r) \neq \emptyset$ implies $Q\left(y_{j}^{\nu}, \ell_{j} / 2\right) \subset Q(\pi(x), 2 r)$. It follows from (II) that $N_{j} \leq A\left(r / \ell_{j}\right)^{n-1}$. Hence we obtain

$$
\sup \left\{\frac{K \mathcal{X}_{E_{j}}}{K \sigma}(x): \sqrt{|x|} \leq b_{j-1}\right\} \leq A \frac{\sigma_{j}}{\ell_{j}^{n-1}} .
$$

Observe from (2.1) and (3.2) that

$$
\begin{aligned}
\frac{\sigma_{j}}{\ell_{j}^{n-1}} & =\left(\frac{\ell_{j}+\rho_{j}}{\ell_{j}}\right)^{n-1} \frac{\sigma\left(Q\left(y, \ell_{j}+\rho_{j}\right)\right)}{\left(\ell_{j}+\rho_{j}\right)^{n-1}}-\left(\frac{\ell_{j}-\rho_{j}}{\ell_{j}}\right)^{n-1} \frac{\sigma\left(Q\left(y, \ell_{j}-\rho_{j}\right)\right)}{\left(\ell_{j}-\rho_{j}\right)^{n-1}} \\
& \rightarrow 0 \text { as } j \rightarrow+\infty .
\end{aligned}
$$

This together with (3.6) concludes (i).

Taking $x=0$ in (i), we obtain

$$
\sigma\left(E_{j}\right)=\sigma(S) \frac{K \mathcal{X}_{E_{j}}}{K \sigma}(0) \rightarrow 0 \quad \text { as } j \rightarrow+\infty
$$

Thus (ii) follows.

Proof of Theorem 1.1. In view of Lemma 3.2, taking a subsequence of $j$ if necessary, we may assume that

$$
\frac{K \mathcal{X}_{E_{j}}}{K \sigma}(x) \leq 2^{-j} \text { for } \sqrt{|x|} \leq b_{j-1}
$$


and $\sigma\left(E_{j}\right) \leq 2^{-j}$. Then $\sigma\left(\bigcap_{k} \bigcup_{i \geq k} E_{i}\right)=0$. For $j \in \mathbb{N}$, let

$$
f_{j}(y)= \begin{cases}(-1)^{I_{j}(y)} & \text { if } y \in \bigcup_{1 \leq i \leq j} E_{i}, \\ 0 & \text { if } y \notin \bigcup_{1 \leq i \leq j} E_{i},\end{cases}
$$

where $I_{j}(y)=\max \left\{i: y \in E_{i}, 1 \leq i \leq j\right\}$. Then we see that $f_{j}$ converges $\sigma$-a.e. on $S$ to

$$
f(y)= \begin{cases}(-1)^{I(y)} & \text { if } y \in \bigcup_{i \geq 1} E_{i} \backslash \bigcap_{k} \bigcup_{i \geq k} E_{i}, \\ 0 & \text { if } y \notin \bigcup_{i \geq 1} E_{i} \text { or } y \in \bigcap_{k} \bigcup_{i \geq k} E_{i},\end{cases}
$$

where $I(y)=\max \left\{i: y \in E_{i}\right\}$ for $y \in \bigcup_{i \geq 1} E_{i} \backslash \bigcap_{k} \bigcup_{i \geq k} E_{i}$. Also, we have the following:

$$
\left|f_{j}\right| \leq 1, \quad\left|f_{j+1}-f_{j}\right| \leq 2 \mathcal{X}_{E_{j+1}} \text { on } S ; \quad f_{j}=(-1)^{j} \text { on } E_{j} ; \quad K f_{j} \rightarrow K f \text { on } \mathbb{R}^{n} .
$$

Let $T \in O(n)$. By Lemma 3.1, we find $x_{j} \in T \gamma \cap G_{j}$ for each $j \in \mathbb{N}$. Then $a_{j} \leq$ $\sqrt{\left|x_{j}\right|} \leq b_{j}$ and $Q\left(\pi\left(x_{j}\right), c_{j} / a_{j}\right) \subset E_{j}$. If $j$ is even, then Lemma 2.3(ii) and (3.7) give

$$
\begin{aligned}
\frac{K f}{K \sigma}\left(x_{j}\right) & =\frac{K f_{j}}{K \sigma}\left(x_{j}\right)+\sum_{k \geq j} \frac{K\left(f_{k+1}-f_{k}\right)}{K \sigma}\left(x_{j}\right) \\
& \geq \frac{K f_{j}}{K \sigma}\left(x_{j}\right)-2 \sum_{k \geq j} \frac{K \mathcal{X}_{E_{k+1}}}{K \sigma}\left(x_{j}\right) \\
& \geq 1-\frac{A}{c_{j}}-2^{1-j} .
\end{aligned}
$$

Similarly, if $j$ is odd, then

$$
\frac{K f}{K \sigma}\left(x_{j}\right) \leq-1+\frac{A}{c_{j}}+2^{1-j}
$$

Hence we conclude from (3.2) that

$$
\liminf _{T \gamma \ni x \rightarrow \infty} \frac{K f}{K \sigma}(x)=-1<1=\limsup _{T \gamma \ni x \rightarrow \infty} \frac{K f}{K \sigma}(x) .
$$

Obviously, $u=K f$ is a solution of (1.1) such that $-1 \leq u / K \sigma \leq 1$ on $\mathbb{R}^{n}$. Thus the proof of Theorem 1.1 is complete.

\section{REMARK}

Our construction and estimates in Sections 2 and 3 are applicable to show Theorem B. Suppose that an unbounded subset $\Omega$ of $\mathbb{R}^{n}$ satisfies the assumption in Theorem B. Then we find a sequence $\left\{x_{j}\right\}$ in $\Omega$ converging to $e$ at $\infty$ such that

$$
\lim _{j \rightarrow+\infty} \frac{\left|x_{j}-\right| x_{j}|e|}{\sqrt{\left|x_{j}\right|}}=+\infty .
$$

Taking a subsequence of $j$ if necessary, we may assume that $\sqrt{\left|x_{j-1}\right|}\left|\pi\left(x_{j}\right)-e\right| \leq 1$. Let $\omega_{j}=\left\{T_{e}\left(x_{j}\right): T_{e} \in O(n)\right.$ preserves $\left.e\right\}$ and let $\omega=\bigcup_{j} \omega_{j}$. Note that $\omega$ is a subset of $\Omega$ 
converging to $e$ at $\infty$. Let $a_{j}=b_{j}=\sqrt{\left|x_{j}\right|}$ and define

$$
\ell_{j}=\frac{\left|\pi\left(x_{j}\right)-e\right|}{3}, \quad c_{j}=\sqrt{a_{j}\left|\pi\left(x_{j}\right)-e\right|} \quad \text { and } \quad \rho_{j}=\frac{c_{j}}{a_{j}}
$$

in place of (3.1). Then these satisfy (3.2) and $3 \ell_{j} \leq 1 / b_{j-1}$. Let $M_{j}, G_{j}$ and $E_{j}$ be as in (3.3), (3.4) and (3.5) respectively. Then the conclusions in Lemma 3.2 hold in this setting as well. Note that $\omega_{j}$ and $G_{j}$ lie on the sphere of center at the origin and radius $\left|x_{j}\right|$. Let $T \in O(n)$. Since $\left\{y \in S:|y-T e|=3 \ell_{j}\right\} \subset \pi\left(T \omega_{j}\right)$, we see that $\pi\left(T \omega_{j}\right) \cap M_{j} \neq \emptyset$, and so $T \omega_{j} \cap G_{j} \neq \emptyset$. Hence we observe the existence of $f$ such that

$$
\liminf _{T \omega \ni x \rightarrow \infty} \frac{K f}{K \sigma}(x) \neq \limsup _{T \omega \ni x \rightarrow \infty} \frac{K f}{K \sigma}(x) \quad \text { for every } T \in O(n) .
$$

Thus $K f / K \sigma$ admits no limits as $x \rightarrow \infty$ along $\Omega(y)$ for every $y \in S$.

\section{REFERENCES}

[1] H. Aikawa, Harmonic functions having no tangential limits, Proc. Amer. Math. Soc. 108 (1990), no. 2, 457-464.

[2] __ Harmonic functions and Green potentials having no tangential limits, J. London Math. Soc. (2) 43 (1991), no. 1, 125-136.

[3] R. Berman and D. Singman, Boundary behavior of positive solutions of the Helmholtz equation and associated potentials, Michigan Math. J. 38 (1991), no. 3, 381-393.

[4] F. T. Brawn, The Martin boundary of $\left.R^{n} \times\right]$ 0, 1, J. London Math. Soc. (2) 5 (1972), 59-66.

[5] P. Fatou, Séries trigonométriques et séries de Taylor, Acta Math. 30 (1906), 335-400.

[6] K. Gowrisankaran and D. Singman, Thin sets and boundary behavior of solutions of the Helmholtz equation, Potential Anal. 9 (1998), no. 4, 383-398.

[7] K. Hirata, Sharpness of the Korányi approach region, Proc. Amer. Math. Soc. 133 (2005), no. 8, 23092317.

[8] A. Korányi, Harmonic functions on Hermitian hyperbolic space, Trans. Amer. Math. Soc. 135 (1969), 507-516.

[9] A. Korányi and J. C. Taylor, Fine convergence and parabolic convergence for the Helmholtz equation and the heat equation, Illinois J. Math. 27 (1983), no. 1, 77-93.

[10] J. E. Littlewood, On a theorem of Fatou, J. London Math. Soc. 2 (1927), 172-176.

[11] A. Nagel and E. M. Stein, On certain maximal functions and approach regions, Adv. in Math. 54 (1984), no. $1,83-106$.

[12] J. Sueiro, On maximal functions and Poisson-Szegö integrals, Trans. Amer. Math. Soc. 298 (1986), no. 2, 653-669.

Department of Mathematics, Hokkaido University, SAPPORo 060-0810, JAPAN

E-mail address: hirata@math.sci.hokudai.ac.jp 